WSRC-TR-91-231, REV. 1

\title{
CHRONIC TOXICITY EVALUATION OF \\ SIMULATED DWPF EFFLUENT TO CERIODAPHNIA DUBIA
}

Report To:

WSRC-TR- $-91-231-$ Rey. 1

DE92 009425

WESTINGHOUSE SAVANNAH RIVER COMPANY

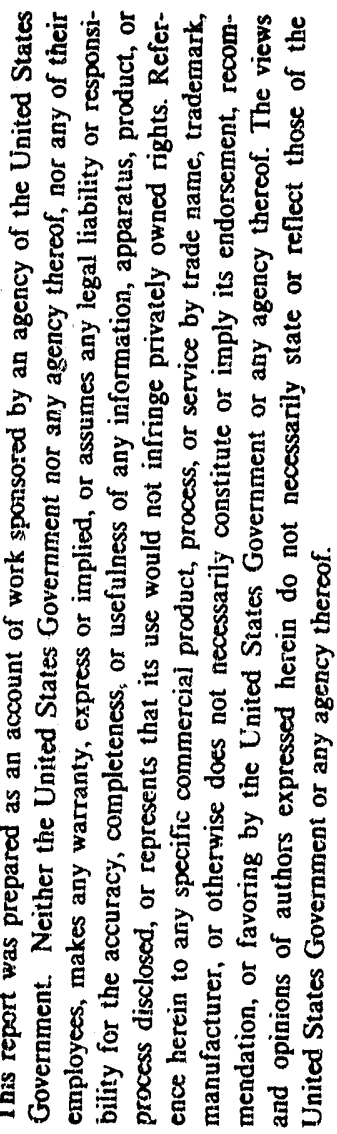

Savannah River site

Aiken, South Carolina

Aprid 1990

\author{
Submitted By: \\ SHEALY ENVIRONMENTAL SERVICES, INC. \\ 400 Graymont Âvenue \\ Columbia, South Carolina \\ (803) 254-9915
}

SCDHEC Laboratory Certification No. 26103

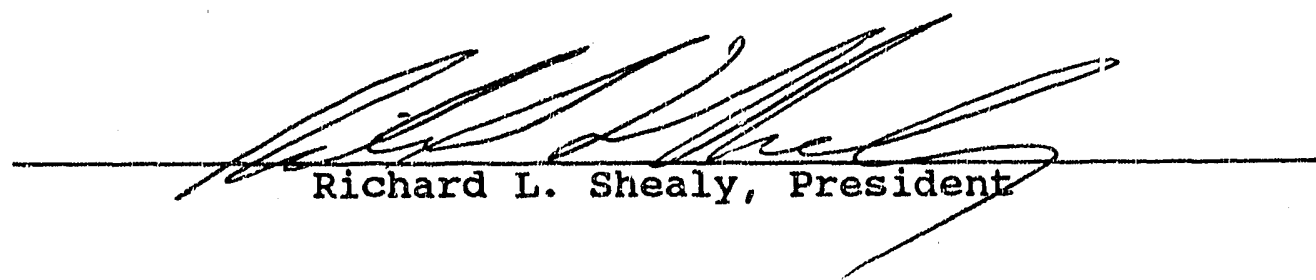


I. INTRODUCTION

A 7-Day Full Series Chronic Toxicity test was conducted April 13

- 20, 1990, for the Savannah River site to assess the chronic toxicity of simulated DWPF effluent to ceriodaphnia dubia.

\section{METHODS}

Dilution water for the toxicity test was collected April 11, 1990 (Lab I.D. No. 90-160I) from McQueens Branch on the Savannah River Site by SHEALY ENVIRONMENTAL SERVICES, INC. personnel and transported iced to the laboratory. The water was filtered with a plankton net $(37 \mathrm{~mm})$ prior to use in the test. Ceriodaphnia for the chronic test had been cultured in Upper Three Runs Creek water since October 25, 1988. Water from Upper Three Runs Creek for the cultures was collected at the northside of a bridge on Road 2-1 on the Savannah River site by SHEALY ENVIRONMENTAL SERVICES, INC. personnel.

All organisms used in the toxicity tests were from SHEALY ENVIRONMENTAL SERVICES, INC.'s in-house cultures which were obtained from the USEPA Newton Laboratory April 20, 1987, Lab I.D. No. 87-271. Ceriodaphnia from in-house cultures are identified and preserved monthly. standard toxicant tests with the EPA reference toxicant cadmium chloride and laboratory reagent grade cadmium chloride are performed monthly on Ceriodaphnia cultured in water from Upper Three Runs creek and in conjunction with the chronic toxicity tests. The results of these tests $\left(48\right.$-Hour central Tendency at $20^{\circ} \mathrm{C}$ was $0.23 \mathrm{ppm}$ ) 
demonstrated that the condition of the cultures were within the acceptable range for the test organisms ( \pm 2 S.D.).

Test methods conformed to those described in USEPA (1989; see Table 1). The 7-day chronic toxicity bioassay was performed April $13-20,1990$, as six treatments exposing 20 test organisms each. The first treatment was the control (100\% filtered McQueens Branch water). The simulated DWPF solutions were $10 \%$, 20\%, 40\%, 70\%, and 100\%. All test solutions were prepared from DETF solutions prepared daily by dosing the dilution water with appropriate aliquots of simulated DWPF (Table 2). The test organisms were exposed to each treatment in individual test chambers. Test solutions were renewed daily.

Dissolved oxygen, water temperature, $\mathrm{pH}$ and conductivity measurements were made daily in conjunction with the test. Temperature was maintained at $25^{\circ} \pm 1^{\circ} \mathrm{C}$ in all test chambers during the test.

The test organisms were placed singly in the test vessels each containing $15 \mathrm{ml}$ of solution. The organisms were between $16-24$ hours old at the start of the test. Transfer of the neonates was accomplished using an eye dropper where the organisms were never removed from solution. All Ceriodaphnia were fed the green alga Selenastrum capricornutum at a rate of approximately 250,000 cells per $\mathrm{ml}$. test solution per day and $0.1 \mathrm{ml}$ Yeast-CerophylTrout chow mixture. Selenastrum cultures were obtained from 
Table 1: Summary of Test Conditions for the Chronic Toxicity Bioassay on DWPF Simulated Effluent with Ceriodaphnia dubia.

1. Temperature:

2. Light Intensity:

3. Photoperiod:

4. Size of test vesse]:

5. Volume of test solution:

6. Age of test organisms:

7. No. animals per test vessel:

8. No. replicate test vessels per concentatration:

9. Total no. organisms per concentration:

10. Feeding regime:

11. Aeration:

12. Dilution water:

13. Test duration:

14. Effects measured:

15. Test acceptability:
$25 \pm 1^{\circ \mathrm{C}}$

Ambient Laboratory Levels

$16 \mathrm{~h}$ light/8 h dark

1 ounce solo plastic disposable cups

$15 \mathrm{ml}$

2-24 hour neonates and all released within the same 8 hour period

1

20

20

Selenastrum capricorrutum at the rate of Approx. 250,000 cells per ml test solution per day and $0.1 \mathrm{ml}$ YCT mixture

None

McQueens Branch

Until 60\% control females have produced 3 broods

Mortality - no movement of appendages on gentle prodding and number of offspring produced

$80 \%$ or greater survival and and average of 15 or more young/surviving female in the control solutions. At least 60\% of surviving females in control should have produced their third brood. 
Table 2. Daily Preparations of simulated DWPF Effluent

$\begin{array}{cccccccc}g / 1 & \text { Initial } & 1 & 2 & 3 & 4 & 5 & 6 \\ \text { Sodium nitrate } & 0.010 & 0.011 & 0.010 & 0.011 & 0.010 & 0.010 & 0.011 \\ \text { Sodium oxalate } & 0.0292 & 0.0310 & 0.0295 & 0.0305 & 0.0291 & 0.0309 & 0.0298 \\ \text { Sodium formate } & 0.0450 & 0.0453 & 0.0449 & 0.0455 & 0.0450 & 0.0449 & 0.0452 \\ \text { Corros. Inib. } & 0.0391 & 0.0384 & 0.0385 & 0.0390 & 0.0385 & 0.0382 & 0.0394\end{array}$

Sodium hypochlorite - weighed $0.01 \mathrm{~g} / \mathrm{l}$ then diluted 100 times $1 \mathrm{ml} \rightarrow->100 \mathrm{ml}$ volumetric flask. 
Carolina Biological supply company and cultured in natural spring water and Alga-Gro media in 1-liter cotton-plugged Erlenmeyer flasks and maintained under bright fluorescent lighting for 6 days. Test chambers here incubated for temperature control with photoperiod held at 16 hours of light and 8 hours of darkness. Randomization of test animals in the incubator and order of feeding was established based on random number tables.

The results of the 7-day chronic toxicity test are given in Table 3. Mortality of the adult females occurred in the 10\%" (0\% mortality), 20\% ( $5 \%$ mortality), $40 \%$ ( $5 \%$ mortality), $70 \%$ ( $10 \%$ mortality), and 100\% (5\% mortality) simulated DEWF effluent concentrations. Five percent $5 \%$ mortality occurred in the control.

Average reproduction and mortality of the adult femailes in the control and simulated DWPF solutions was as follows:

\section{Offspring per Female}

Control

DWPF

Solutions

$10 \%$

$20 \%$

$40 \%$

$70 \%$

$100 \%$
$=20.6$

\section{Mortality}

$5 \%$

$=20.7 \quad 0 \%$

$=19.0 \quad 5 \%$

$=18.6 \quad 5 \%$

$\begin{array}{ll}=19.8 & 10 \%\end{array}$

$=22.9 \quad 5 \%$ 
Table 3. Reproduction/Mortality Data recorded in conjunction with the 7-Day chronic toxicity test

6 with Ceriadaphnia Dubia for Hestinghouse Savannah River Company on simulated DWPF effluent.

Control

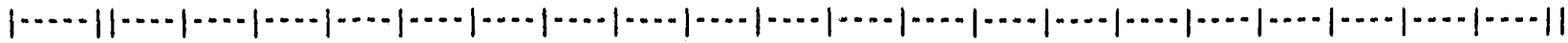

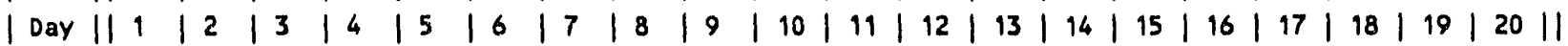

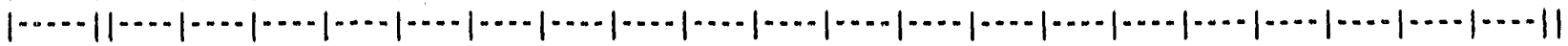

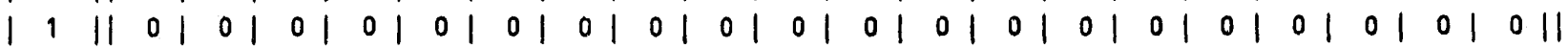

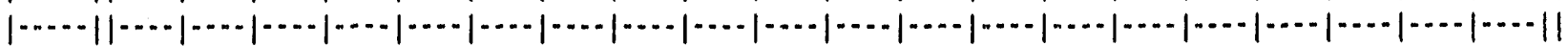

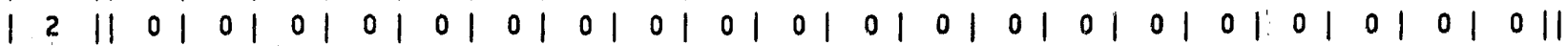

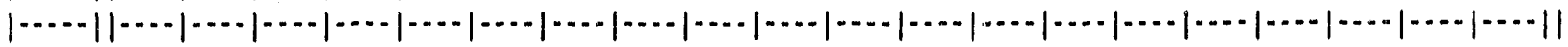

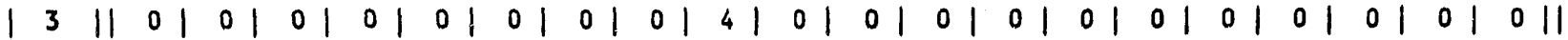

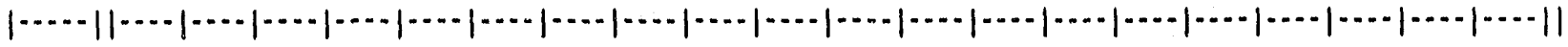

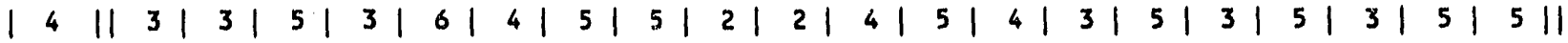

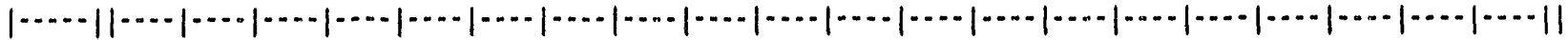

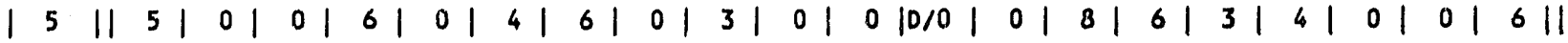

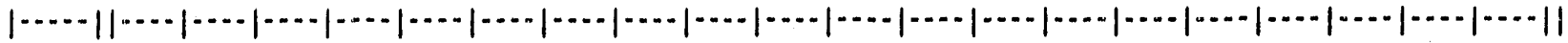
$|6||10| 9|8| 0|9| 11|5| 5|10| 7|10| \cdot|11| 10|9| 0|11| 12|3| 6||$

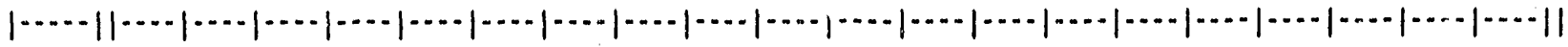
$|7||4| 10|9| 12|12| 0|5| 14|8| 11|10|-|3| 0|0| 12|3| 5|4| 9 \mid 1$

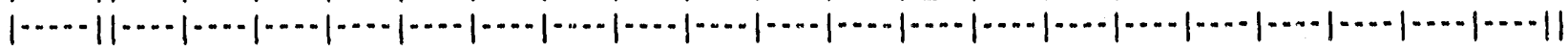
|TOTALI| 22 | 22 | 22 | $21|27| 19|21| 24|27| 20|24| 5|18| 21|20| 18|23| 20|12| 26$ ||

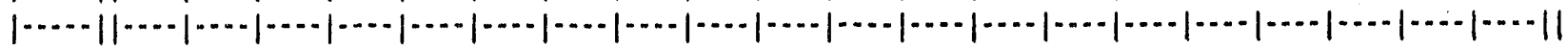

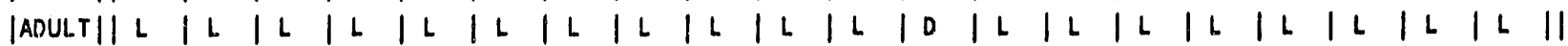

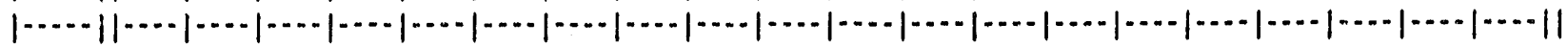

L=Live

$D=$ cead

$$
\bar{x}=20.6
$$

Treatment $1(10 \%)$

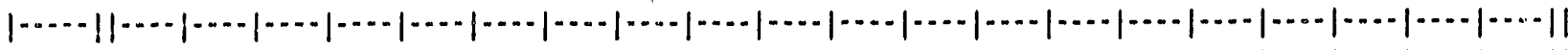

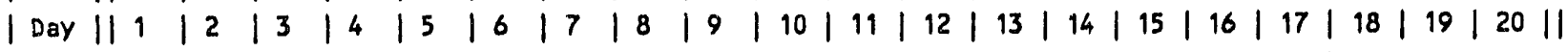

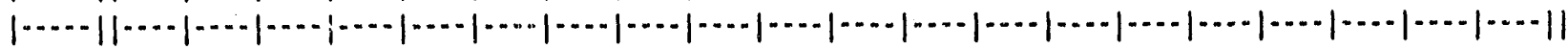

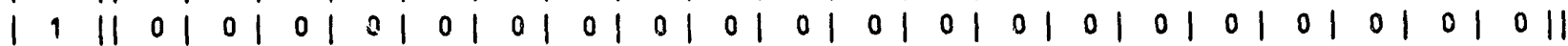

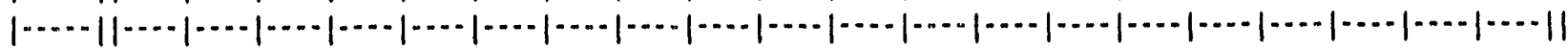

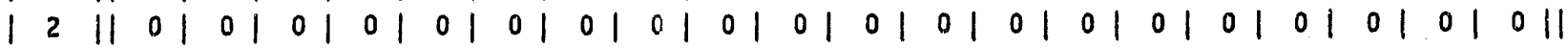

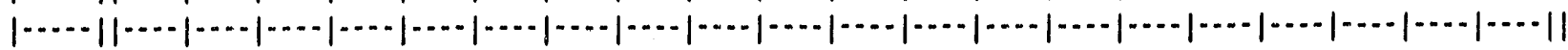

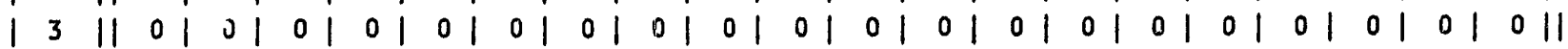

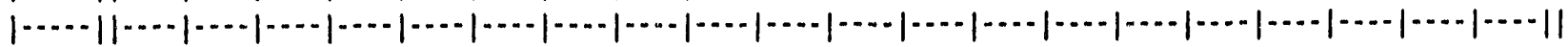

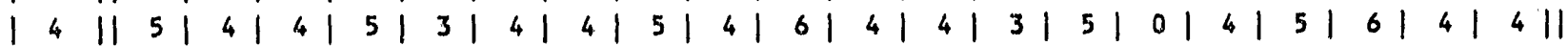

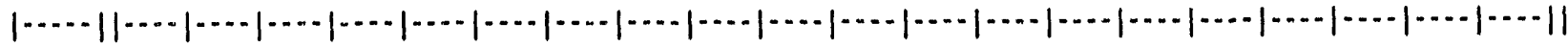

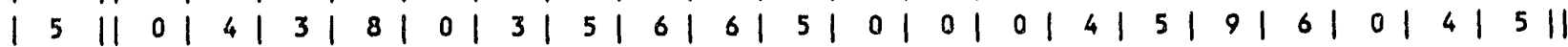

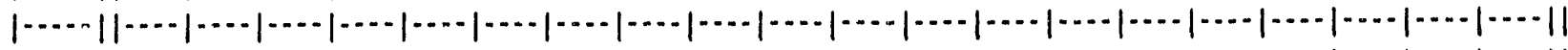
$|6||11| 10|9| 10|3| 14|10| 0|0| 9|7| 10|11| 10|4| 12|0| 9|9| 8||$

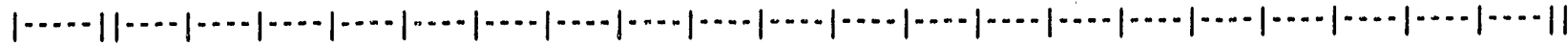

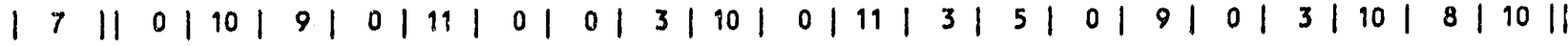

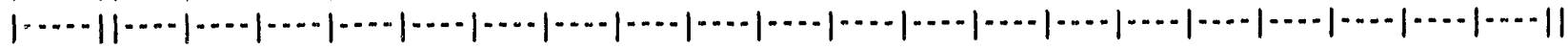
|TOTALI| $16|28| 25|23| 17|21| 19|14| 20|20| 22|17| 19|19| 18|25| 14|25| 25 \mid 27$ ||

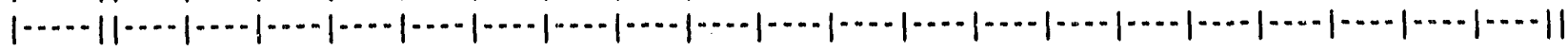
|ADULTIIL IL IL IL IL IL IL IL IL IL IL IL IL IL IL IL IL IL IL IL II

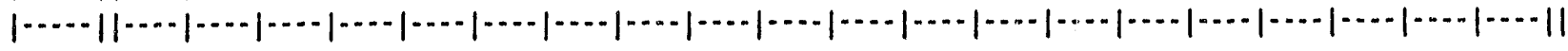
L=Live D=Dead $\bar{x}=20.7$ 
Table 3. Reproduction/Mortality Data recorded in conjunction with the 7-Day chronic toxicity test

Treatment 2 (20\%)

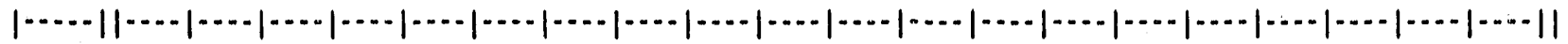

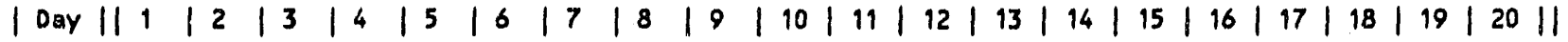

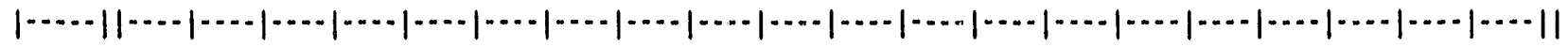

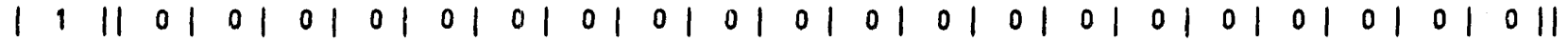

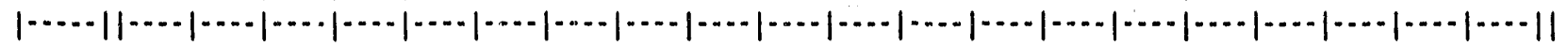

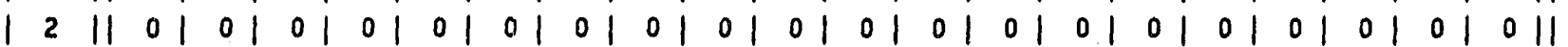

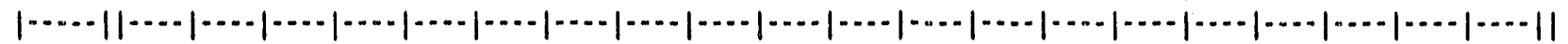

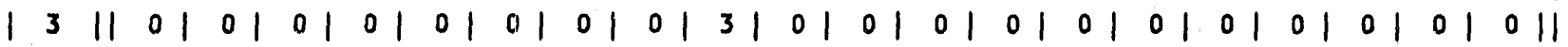

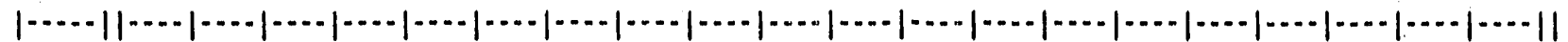

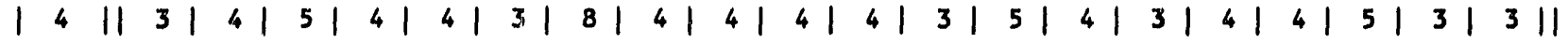

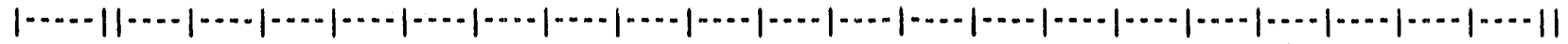

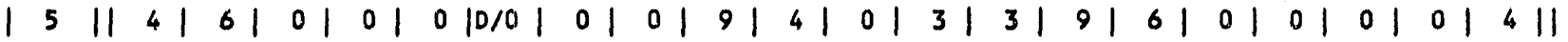

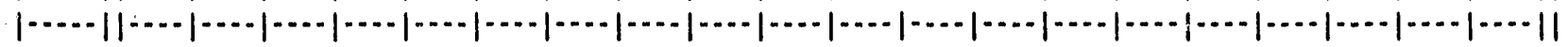

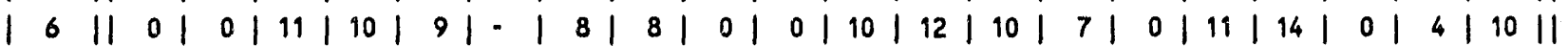

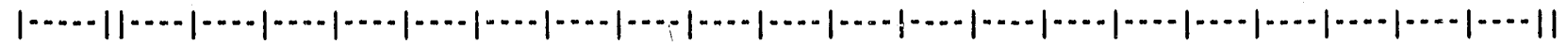

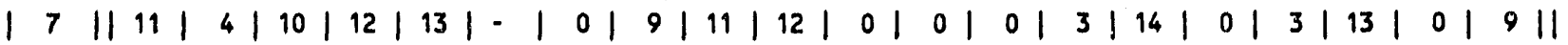

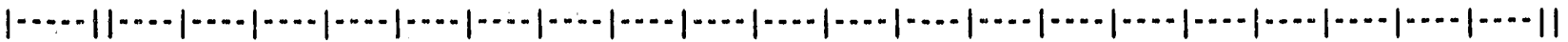
|TOTAL || $18|14| 26|26| 26|3| 16|21| 27|20| 14|18| 18|23| 23|15| 21|18| 7 \mid 26$ ||

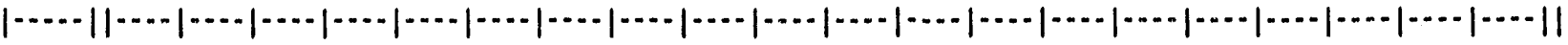
|ADULTIIL IL IL IL IL ID IL IL IL IL IL IL IL IL IL IL IL IL IL IL II

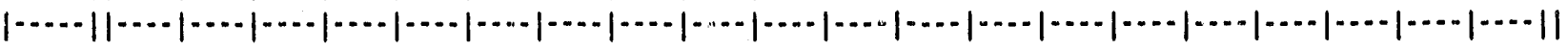
L=Live

D=Dead

$$
\bar{x}=19.0
$$

Treatment 3 (40\%)

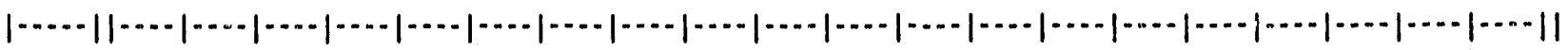

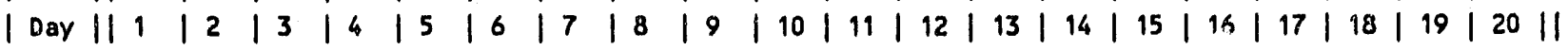

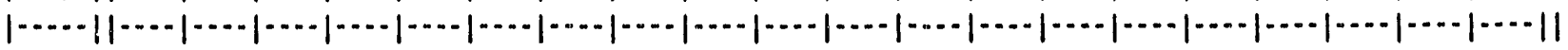

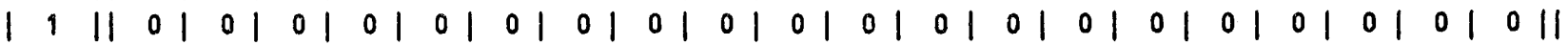

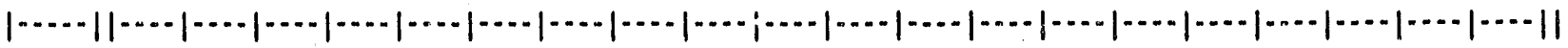
12|| \begin{tabular}{llllllllllllllllll|l|l|l|l|l|l|l|l|l|l}
1 & 0 & 0 & 0 & 0 & 0 & 0 & 0 & 0 & 0 & 0 & 0 & 0 & 0 & 0 & 0 & 0 & 0 & 0 & 1 & 0 & 1 & 0 & 1 & 0 & 1 & 0 & $\|$
\end{tabular}

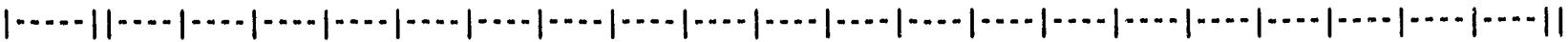

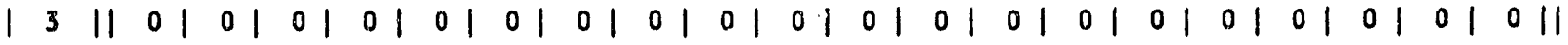

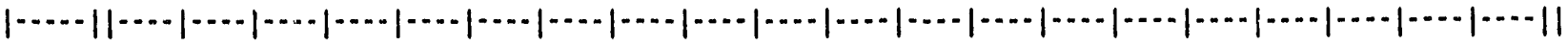
$\mid$\begin{tabular}{l|l|l|l|l|l|l|l|l|l|l|l|l|l|l|l|l|l|l|l|l|l|l} 
& 4 & 4 & 3 & 3 & 6 & 4 & 4 & 5 & 4 & 4 & 5 & 4 & 3 & 2 & 4 & 3 & 4 & 4 & 4 & 5 & 5
\end{tabular}

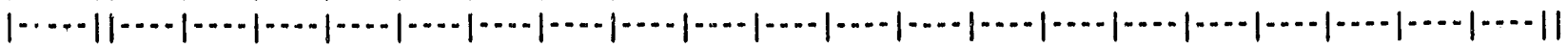

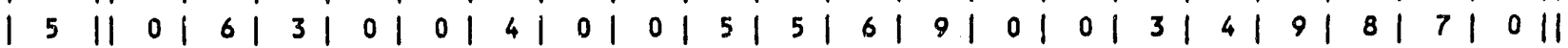

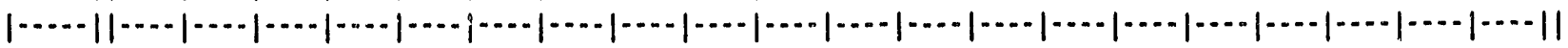

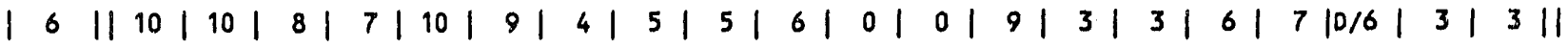

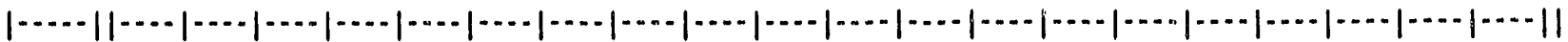

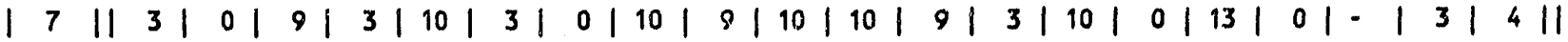

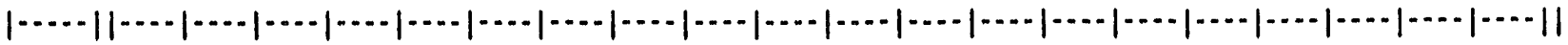
|TOTAL || $17|19| 23|16| 24|20| 9|19| 23|26| 20|21| 14|17| 9|27| 20|18| 18 \mid 12$ ||

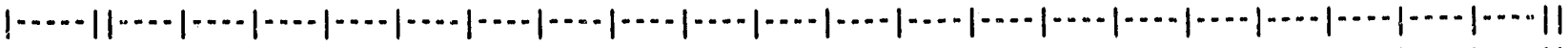
FAOULTIL IL IL IL IL IL IL IL IL IL IL IL IL IL IL IL IL ID IL IL IE

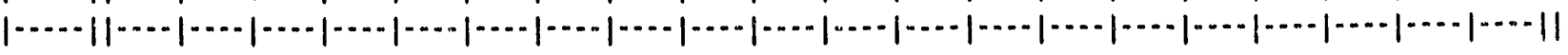
L=Live

$D=$ Dead

$$
\bar{x}=18.6
$$


Table 3. Reproduction/Mortality Data recorded in conjunction with the 7-Day chronic toxicity test with Ceriodaphnia Dubia for Westinghouse Savannah River Company on Simulated DUPF effluent. Treatment 4 (70\%)

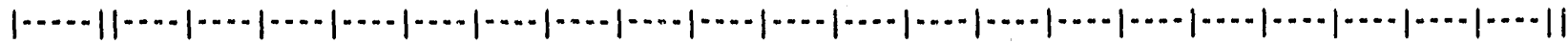

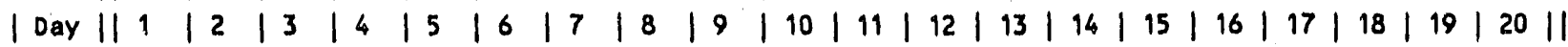

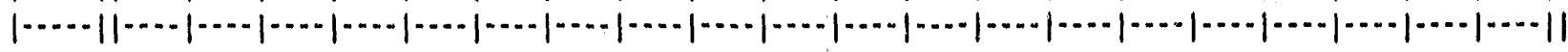

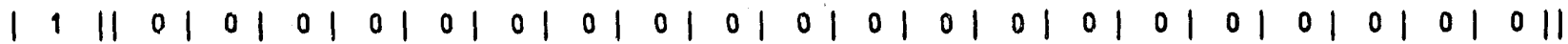

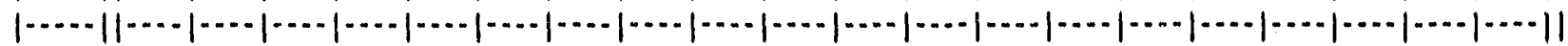

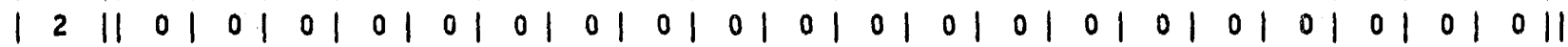

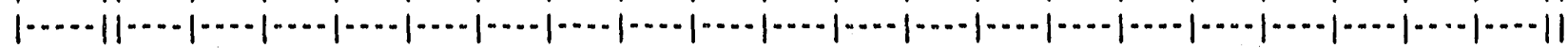

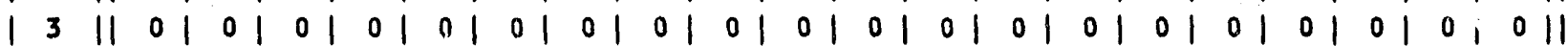

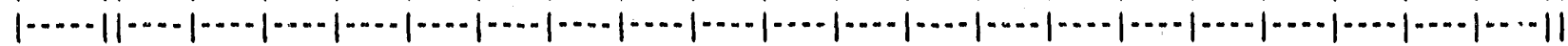
$|4||4| 4|0 / 0| 0|3| 4|6| 3|3| 4|4| 5|2| 4|4| 2|4| 5|4| 6 \mid 1$

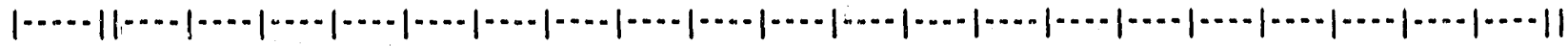

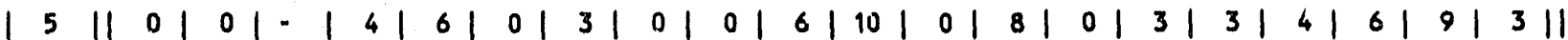

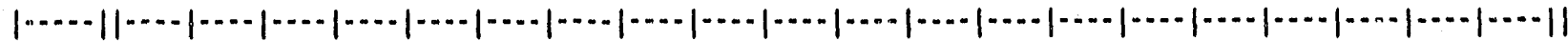

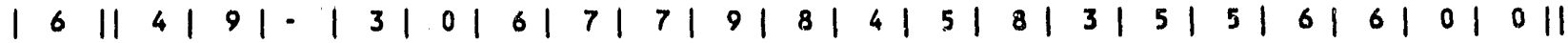

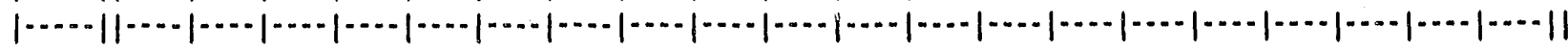
$|7||13| 10|\cdot| 17|10| 10|8| 8|0 / 0| 3|4| 9|0| 4|10| 10|7| 6|11| 14||$

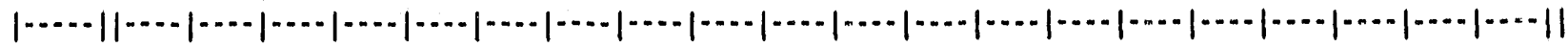
|TOTAL|| $21 \mid 23$ | $0|24| 19|20| 24|18| 12|21| 22|19| 18|21| 22|20| 21|23| 24 \mid 23$ ||

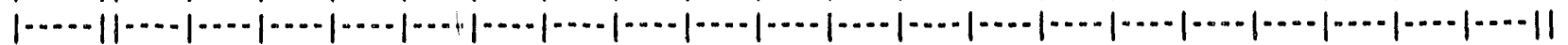

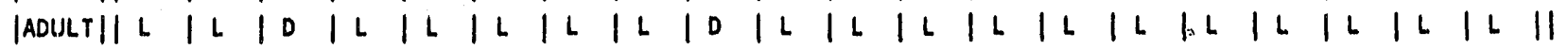

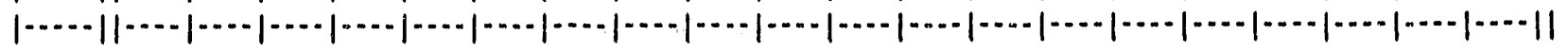
$L=$ Live

D=Dead

\section{$\bar{x}=19.8$}

\section{Treatment 5 (100\%)}

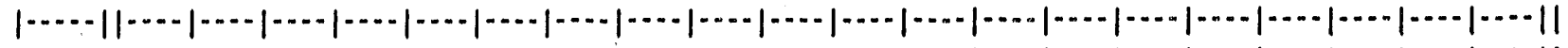

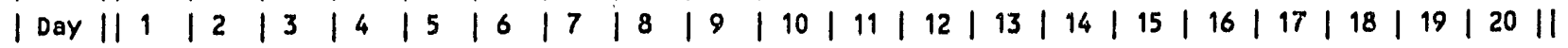

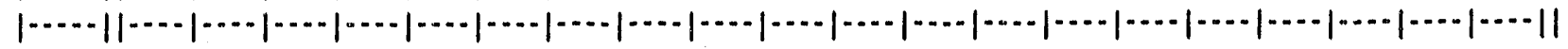

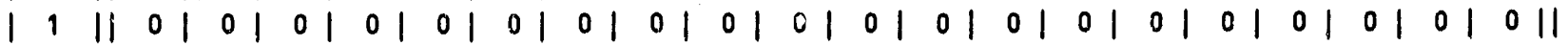

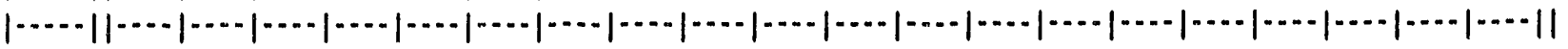

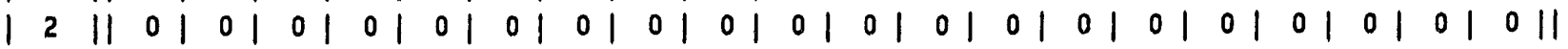

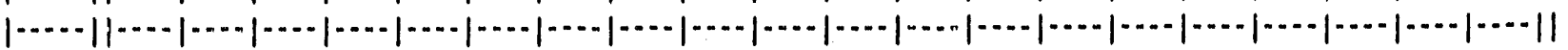

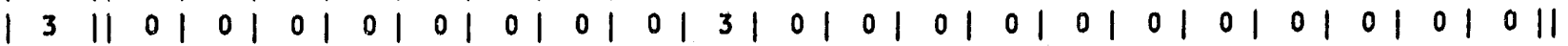

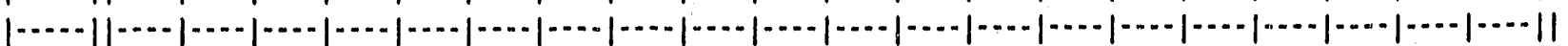
$14|14| 2|4| 3|5| 4|5| 5|4| 3|3| *|4| 6|3| 0|0| 4|4| 6 \mid 1$

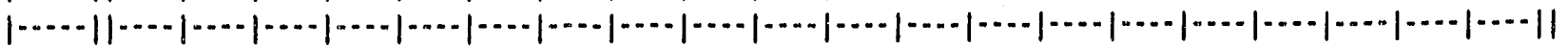

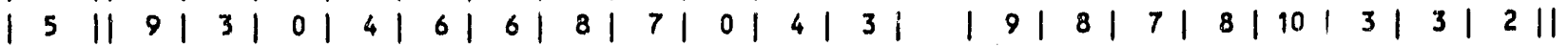

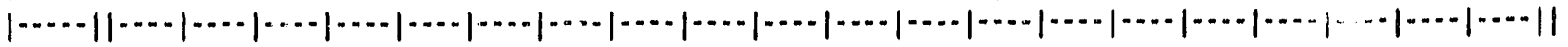
$|6||6| 0|10| 4|14| 0|10| 10|9| 8|14| 0|0| 0|10| 13|0| 0|11| 9 \mid 1$

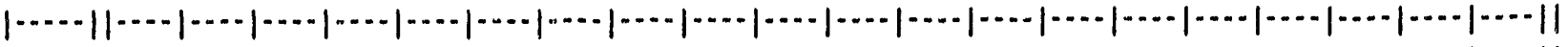
$|7||12| 11|8| 14|0| 16|0| 0 / 0|0| 12|0| \quad|10| 13|10| 3|10| 13|0| 3||$

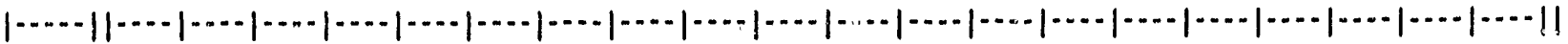
|TOTALI| $31|16| 22|25| 25|26| 23|22| 16|27| 20|*| 23|27| 30|24| 20|20| 18|20| \mid$

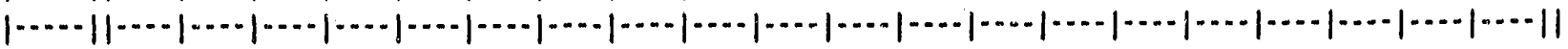

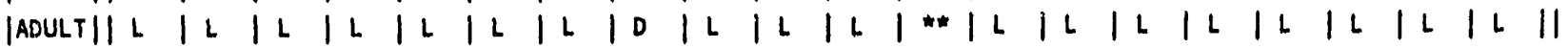

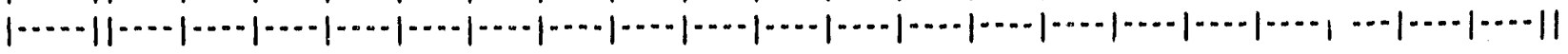
$L=L$ ive $D=$ Dead $\bar{x}=22.9$

*Animal lost during transfer - not included in reproduction data. 
The reproduction data were tested for normality and homogeneity of variances using the Chi-square Goodness of Fit Test and Bartlett's Test, respectively $(p=0.01)$. The data was found to be not normally distributed (Chi-square $=17.36$, Critical value $=$ 13.28), therefore, the non-parametric Wilcoxon's Rank Sum Procedure was used. Results from this test indicated no chronic toxicity at any of the concentrations tested at the probability level of 0.05 (Appendix A). The no observed effect concentration (NOEC) was the 100\%. Water chemistry data taken in conjunction with the chronic toxicity test are given in Table 4. All parameters monitored were within acceptable limits for bioassay purposes. 
Table 4: Water Chemisty Data recorded in conjunction with the 7-Day Chronic Toxicity Test with

Ceriodaphnia dubia for WESTINGHOUSE SAVANNAH RIVER COMPANY on simulated DWPF eifluerit.

DAY 1 DAY 2 DAY 3 DAY 4 DAY 5 DAY 61 UAY 711 Conc. | Parameter ||Init.| old |renew| old |renew| old |renew| old |renew| old |renew| old |renew| old |renew||

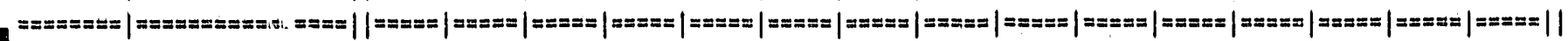

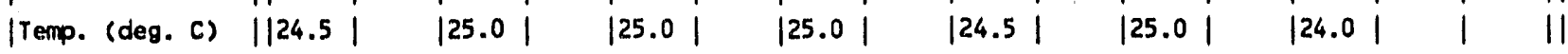

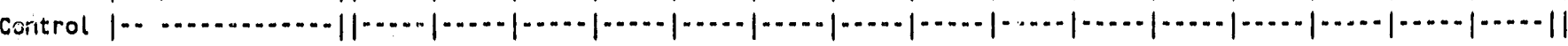

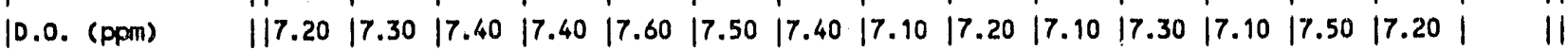

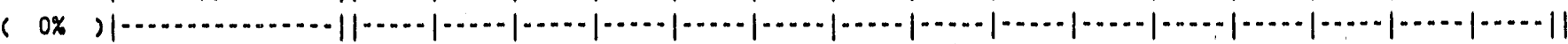

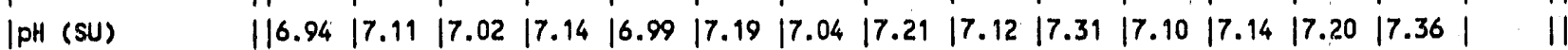

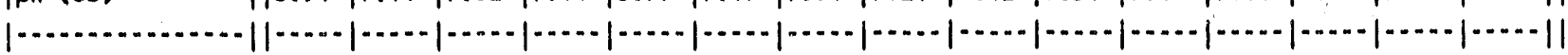

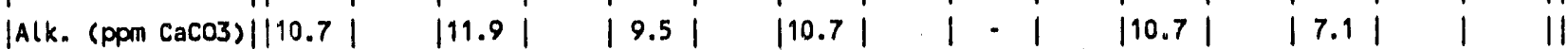

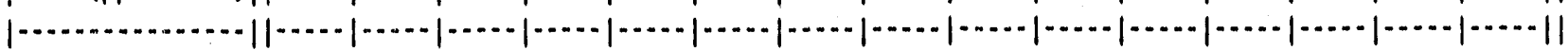
|Hard.(ppon Caco3)||15.7| |23.5 | |19.6| |15.7| |19.6| |19.6| |15.7| | ||

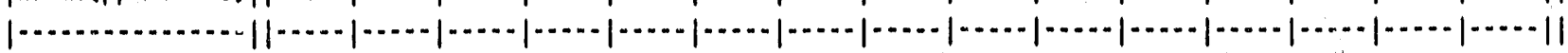
|Cond. (umhos $/ \mathrm{cm})|| 54.0|\quad| 54.0|\quad| 54.0|\quad| 53.0|\quad 154.0| \quad|53.0| \quad|53.0| \quad|\quad| \mid$

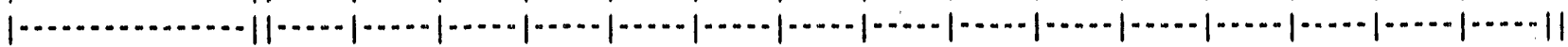

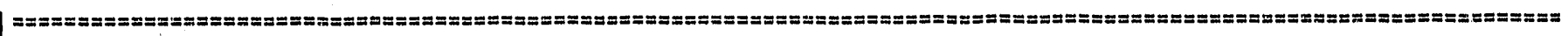

DAY 1 DAY 2 DAY 3 DAY 4 DAY 5 DAY 6 DAY 7 II Conc. I Parameter |linit.| old |renew| old |renew| old |renew| old |renew| old |renew| old |renew| old |renew|l

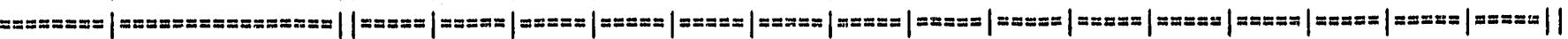

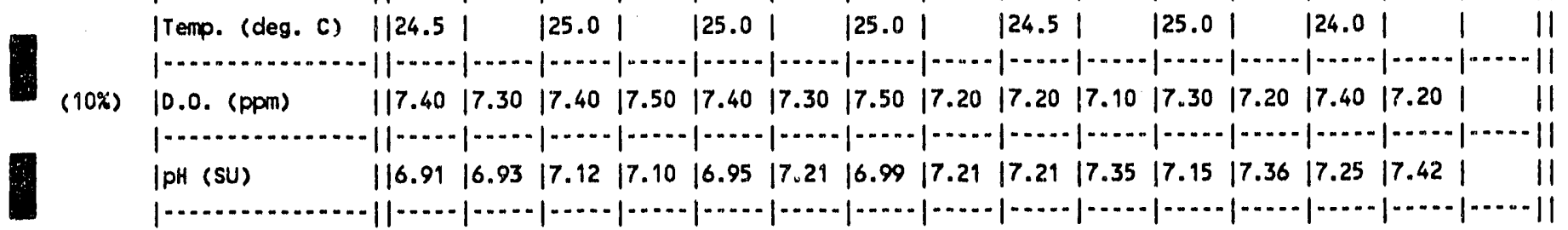

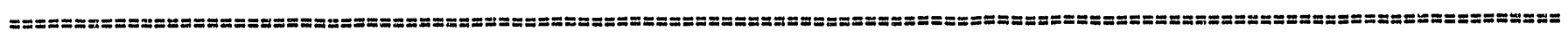

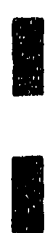

1 DAY 1 DAY 21 DAY 31 DAY 4 DAY 51 DAY 61 DAY 711 Conc. I Parameter |linit.| old |renew| old |renew| old |renew| old |renew| old |renew| old |renew| old |renew|l

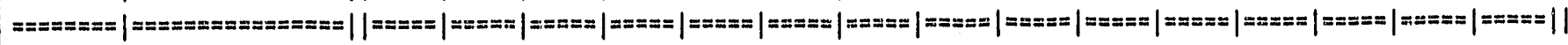 |Temp. (deg. C) || $24.5 \mid$
$|25.0|$
$|25.0|$
$|25.0|$
$|24.5|$
$|25.0|$
$|24.0| \quad|1|$

(20\%)

|

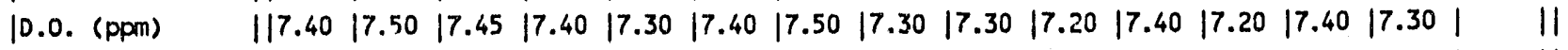

|

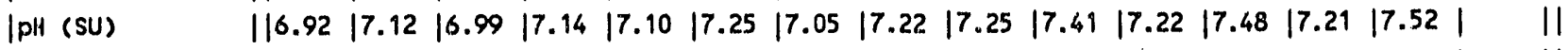

|

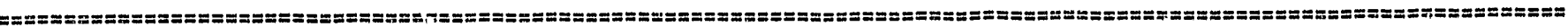


Table 4. Water Chemisty Data recorded in conjunction with the 7-Day Chronic Toxicity Test with

Ceriodaphnia dubia for MESTIMGHOJSE SAVANNAH RIVER COMPANY on simulated OWPF effluent.

DAY 1 DAY 21 DAY 3 DAY 4 DAY 5 DAY 6 I DAY 7 II

Conc. I Parameter ||init.| old |renew| old |renew| old |renew| old |renew| old |renew| old |renew| old |renew||

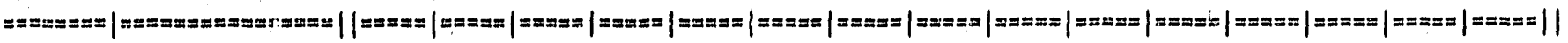

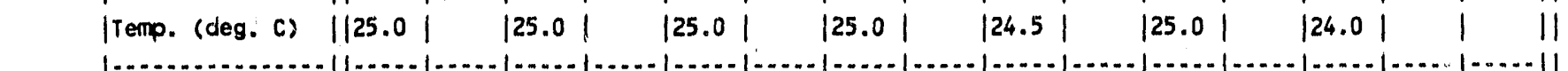

(40\%) $\quad$ |0.0. (ppm) $\quad|17.70| 7.60|7.70| 7.20|7.60| 7.40|7.40| 7.20|7.20| 7.10|7.30| 7.30|7.50| 7.40 \mid$

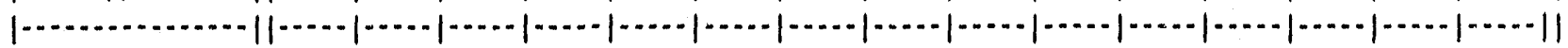

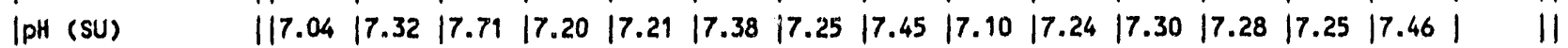

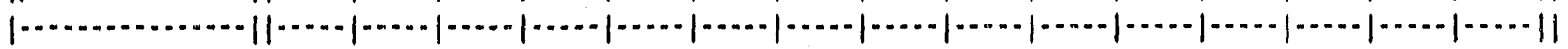

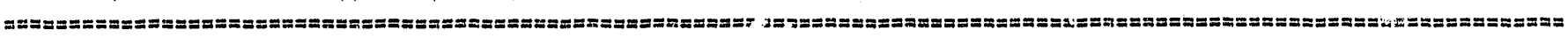

\section{DAY 1 DAY $2 \mid$ DAY 3 DAY 4 DAY $5 \mid$ DAY 6 DAY 711}

Conc. I Paranetiar |linit.| old |renew| old |renew| old |renew| old |renew| old |renew| old |renew| old |renew|l z=z=z=s=| $\mid$ remp. (deg. c) || $25.0|\quad| 25.0|\quad| 25.0|\quad| 25.0|\quad| 24.5|\quad| 25.0|\quad| 24.0|\quad| \quad||$

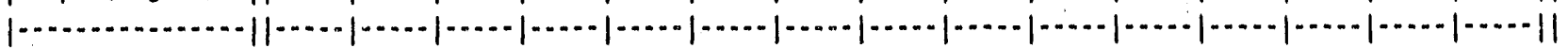

(70\%) |0.0. (ppm) $\quad|7.90| 7.30|7.80| 7.20|7.60| 7.50|7.20| 7.40|7.20| 7.30|7.40| 7.20|7.50| 7.60|| \quad||$

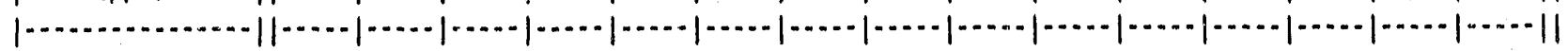

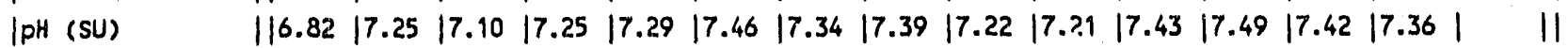
|

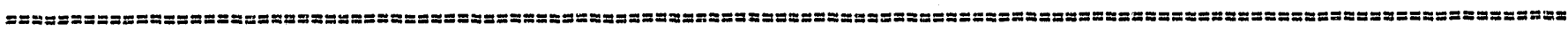

DAY 1 DAY 21 DAY 3 DAY 4 DAY 5 DAY 6 DAY 7 II Conc. I Paraneter |linit.| old |renew| old |renew| old |renew| old |renew| old |renew| old |renew| old |renew|l

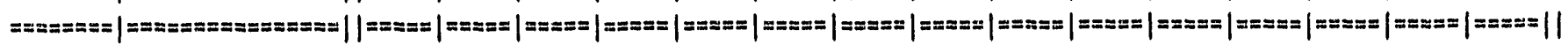

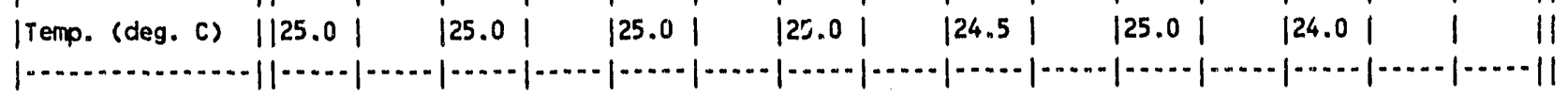

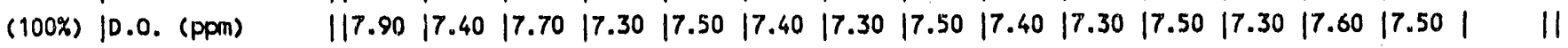
|

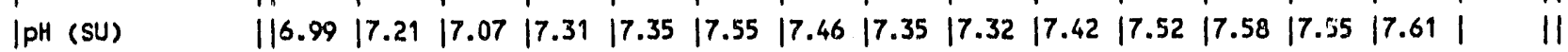

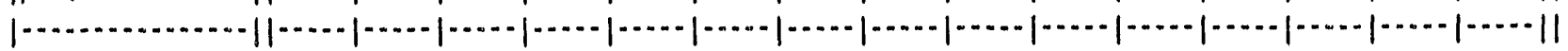
$|A| k .(p p m$ Caco3)||78.2 $|77.0| \quad|75.8| \quad|74.7| \quad|73.5| \quad|77.0| \quad|74.7| \quad|\quad| \mid$

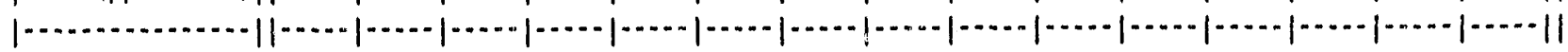
|Hard.(ppm Caco3)||19.6 | |15.7| |19.6| |15.7| |15.7| |11.8| |19.6 | | $1 \mid$

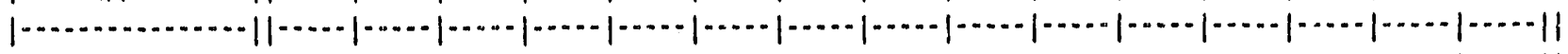

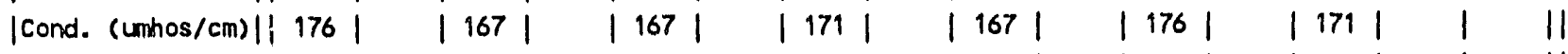

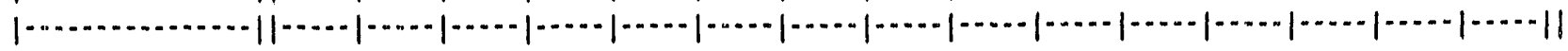

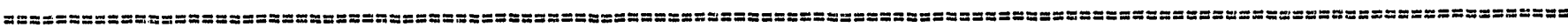


APPENDIX A: Summary of statistical Analyses of Survival and Reproduction Data for the 7-Day Chronic Ceriodaphnia dubia Test for the simulated DWPF effluent.

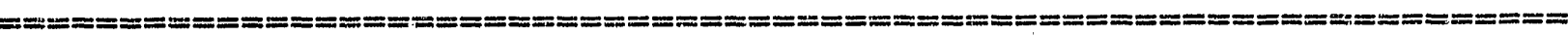

Mortality Data

Fisher's Exact Test: No significant mortality observed at the probability level of 0.05 .

Reproduction Data

Chi-Square Goodness of Fit Test $=\underline{17.36}$

Bartlett's Test for Homogeneity of Variances $=\underline{0.432}$

WILCOXON'S RANK SUM PROCEDURE

CONCENTRATION

10

20

40

60

80
CALCULATED

RANK SUM

$\mathrm{N} / \mathrm{A}$ *

376.5

334.0

391.5

N/A*
CRITICAL

RANK SUM

327

327

327

* Effluent with greater number of offspring than the control. 
然
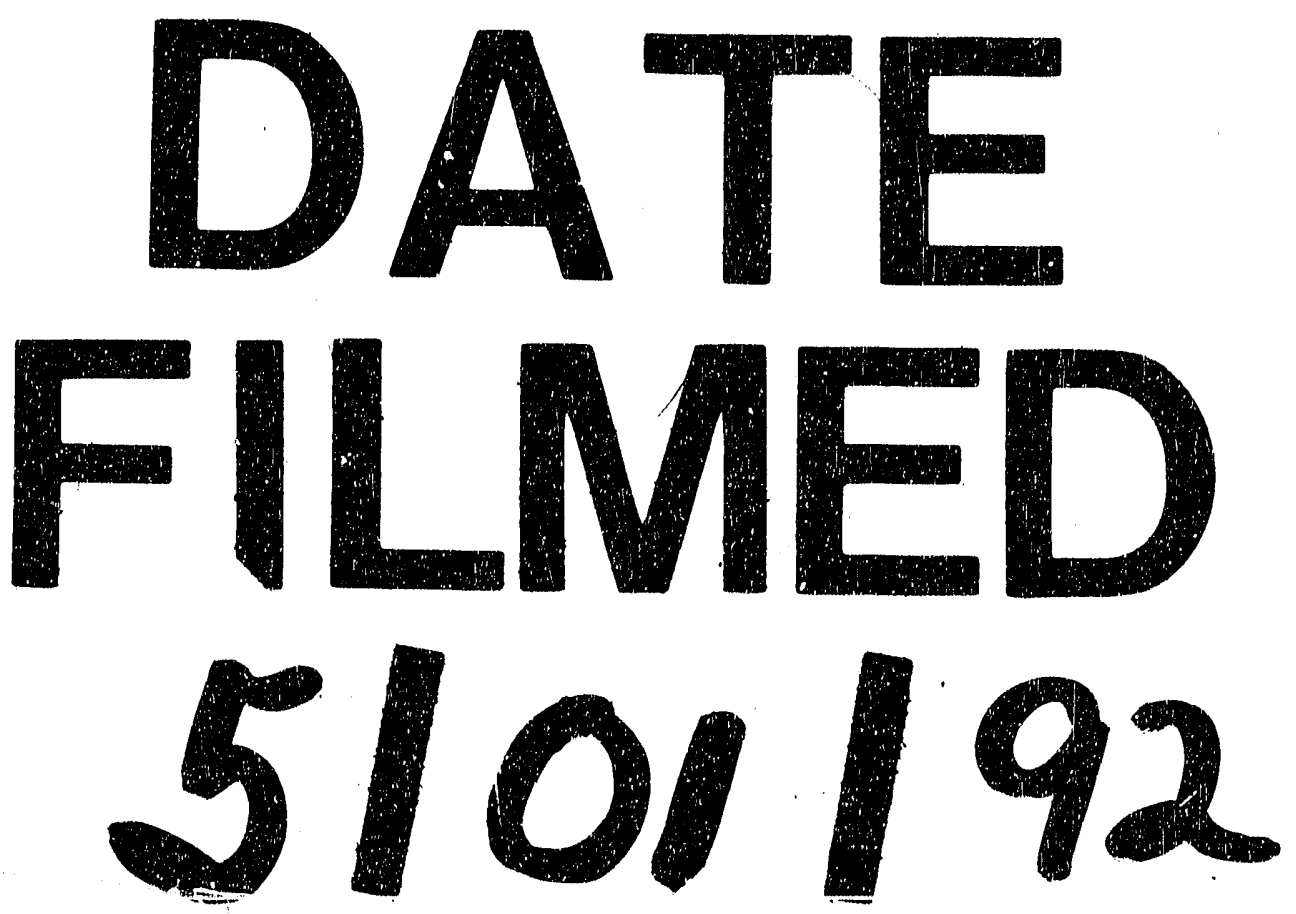
